\title{
Correction of Class II Division 2 Malocclusion with Traumatic deep bite - Non-extraction Therapy
}

\author{
Sultana $\mathrm{S}^{1}$ BDS and Hossain $\mathrm{MZ}^{2}$ BDS, PhD
}

\begin{abstract}
This case report describe the management of a 22 years old male patient having class II div 2 malocclusion with traumatic deep bite. Intraoral examination revealed that patient had lingually inclined maxillary central incisor, labially flared maxillary lateral incisors, exaggerated lower curve of spee, and moderate crowding in lower jaw. Patient also tend to exhibit deep mentolabial sulcus and unaesthetic smile. Anterior flat bite plane was treatment plan for improving deep bite and also Mandibular downward backward rotation and Camouflage nonextraction treatment was decided for this patient because presence of lower crowding which is easy to correction and flare lower incisor for improving overjet and interincisal angle.
\end{abstract}

KEY WORD: Traumatic bite, Non extraction, Camouflage

\section{INTRODUCTION}

A class II division 2 malocclusion is a subdivision of the Angle class II classification and is defined by a class II division 2 incisor relationship, with the incisal edges of the mandibular incisors occluding posterior to the cingulum plateau of the maxillary central incisors, which are retroclined. Typically, there is an increased and complete overbite cause by both dento-alveolar and skeletal factors, also the retroclined labial segments, and reduced Frankfort-mandibular planes angle and lower anterior face height present. A traumatic overbite to the gingiva of the lower labial segment labially or the upper incisors palatally is occasionally seen, which in the presence of poor oral hygiene can result in stripping of the gingival attachment. ${ }^{1}$ The possible etiologic factor is strong vertical posterior development of the mandible with upward and forward rotation and skeletofacial hypodivergence anteroposteriorly, and the lips are typically competent, with a high lower lip line, which may rest on the cervical one-third of the maxillary central incisors. The lower lip also covers a greater surface of the upper central incisors as they erupt, leading to retroclination. ${ }^{2}$ To correct a class II division 2 incisor relationship, overbite reduction is often critical. the overbite can be reduced effectively using removable appliances with a flat anterior bite plane. Alternatively, in the presence of a skeletal class II pattern, a functional appliance may also be helpful to reduce the overbite. ${ }^{3}$ Both of these treatment modalities can allow unimpeded eruption of the molars and premolars. Consequently, the lower face height increases and compensatory growth and adaptation at the condyles stabilizes overbite reduction. Although camouflage may be attempted by extracting premolars, the soft tissue objectives may be impossible to meet. In class II patients with mild to moderate skeletal discrepancies, dental compensation may well be the treatment of choice. Common treatment procedures for such patients including flaring of incisors, interproximal tooth reduction, and extraction. ${ }^{4-6}$

\section{CASE HISTORY AND DIAGNOSIS}

A 22 years old male came to the Orthodontic Department of Dhaka dental College and Hospital for Orthodontic treatment with the chief complain of- Irregularities of teeth, and Upper anterior teeth is flare.

Extra oral examination revealed -his face profile was convex, symmetrical oval face, with competent lip, Deep mento labial sulcus with button like chin. No temporomandibular signs or symptoms were detected or reported (fig-1). Intra oral Examination revealed that patient was in the permanent dentition stage, and good oral hygiene. Class II molar and canine, and Class II div 2 incisor relationship, he had $1 \mathrm{~mm}$ overjet and $6 \mathrm{~mm}$ overbite. Moderate crowding was present in the lower jaw and lingually placed lower left 2nd premolar. There was exaggerated curve of spee (fig-2). Tooth arch discrepancy was $-7 \mathrm{~mm}$ in upper jaw, and $-5 \mathrm{~mm}$ in lower jaw.

Panaromic radiographs revealed that alveolar bone level was normal. Presence of a full complement of permanent teeth except all third molars which was impacted.All teeth appear caries-free, no pathological lesion is seen (fig-3).

\footnotetext{
1.Dr. Sharmin Sultana BDS, FCPS part II trainee, Dept. of Orththodontics and Dentofacial Orthopedics, Dhaka Dental College and Hospital. and 2. Prof. Dr. Md. Zakir Hossain BDS, PhD, Professor and Head, Dept. of Orththodontics and Dentofacial Orthopedics, Dhaka Dental College and Hospital.
} 
Cephalometric evaluation showed that prognathic Maxilla and retrognathic Mandible. Decreased MPA , and increased IIA (fig-5).

\section{TREATMENT OBJECTIVES}

The goal of orthodontic treatment for the patient were

1. To establish normal overbite and overjet.

2. To eliminate dental crowding in lower jaw.

3. To improve inter-incisal angle.

4. To improve aesthetic.

\section{TREATMENT MECHANICS AND PROGRESSION}

Considering all aspects of the case in detail, non extraction treatment plan was established and starting with URA with anterior flat bite plane to reduce the overbite (fig-5). After 3 month of subsequent visit fixed appliance treatment was started in the upper and lower arches (Standard edge wise 0.018' slot bracket). Initial alignment \& levelling of both jaw by using 0.014 " ss round multiloop arch wire with incorporated anterior bite plane was present until place the rigid arch wire in the lower jaw to hold the imrove overbite (fig-6). After 4 months of subsequent visit, placed 0.016” ss round archwire and open coil spring was placed between lower left sided 1st molar and 1st premolar to create space for lingually placed 2nd premolar (fig- 7) . After opening space 0.014' 'niti arch wire was placed to bring the 2nd premolar into normal occlusion, in that time lock was free by using posterior bite plane (fig-8). In upper jaw after proclination of central incisor, placed a 16 X 22," rectangular arch wire with labial crown torque for 3 months. Class II elastic was used for better interdigitation by using rigid arch wire of $16 \mathrm{X} 22$ ', rectangular for 2 months. final finishing and detailing was done by 1 st order bend off-set and inset by using 16 X 22," rectangular arch wire for another 2 months (fig-9). After satisfactory interdigitation and detailing occlusion the fixed appliance were removed and maxillary and mandibular Hawley retainer were placed. The active orthodontic treatment time was 16 months.
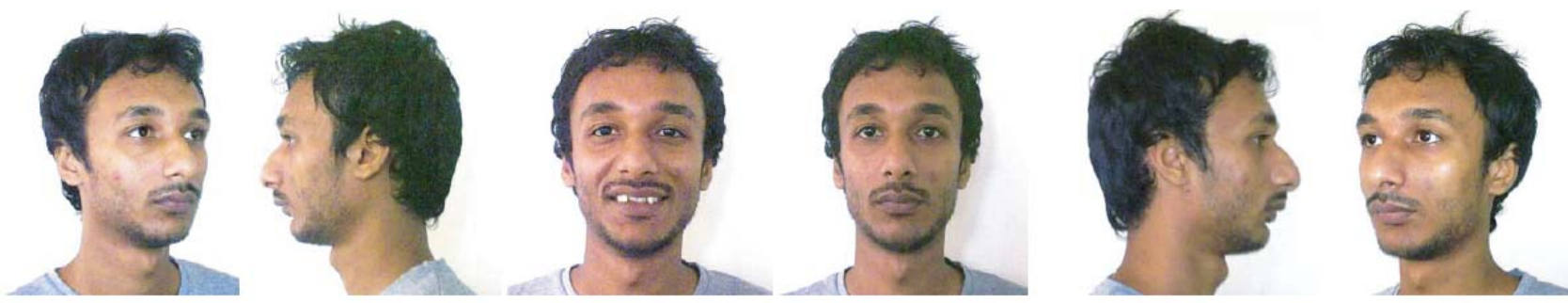

Fig-1 Pretreatment extra oral photographs
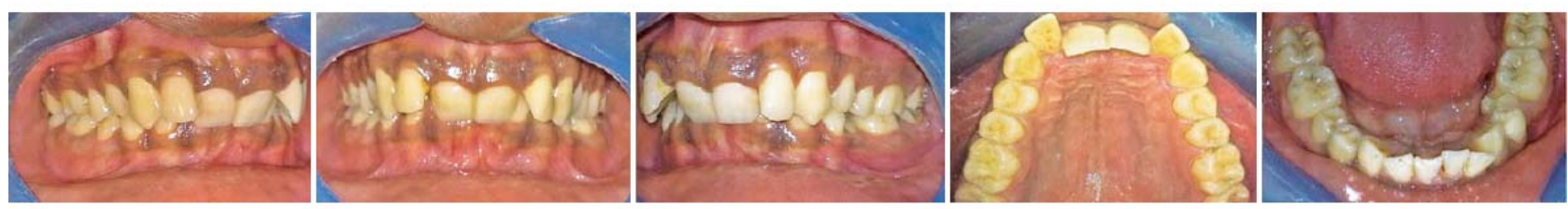

Fig-2 Pretreatment intraoral photographs

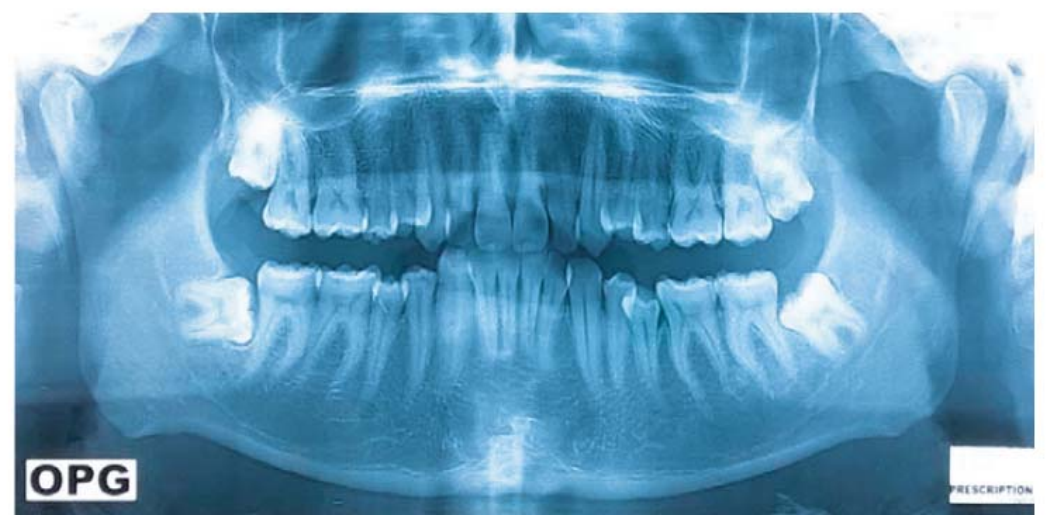

Fig-3 Pre treatment Orthopentomograph (OPG) 


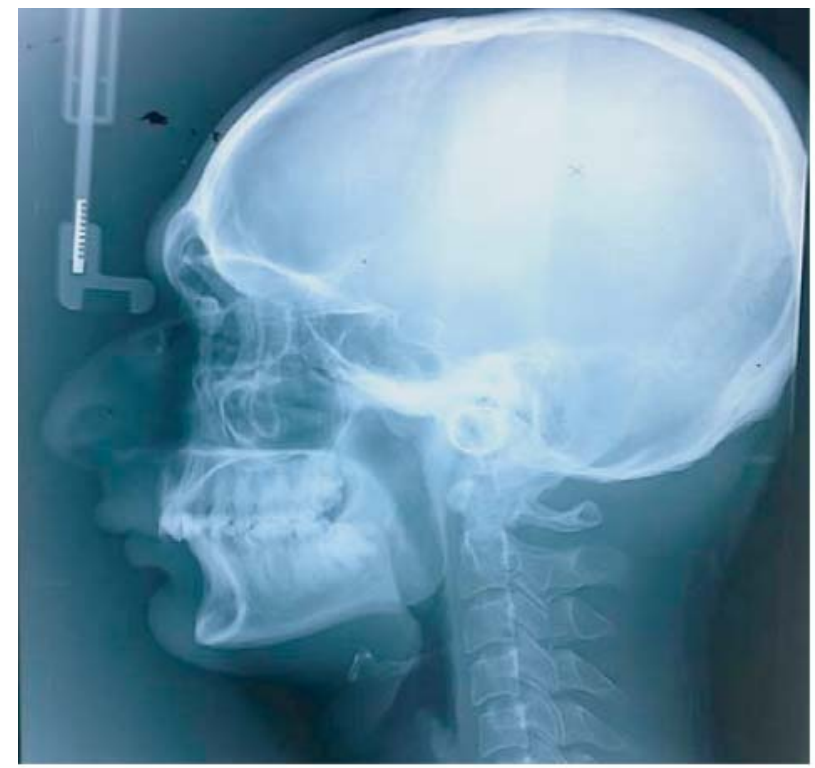

Fig-4 Pre treatment Cephalometric analysis: SNA: $85^{\circ}$ ANB: $10^{\circ} \mathrm{MPA}$ : $24^{\circ}$, U1 to $\mathrm{NA}^{\circ}: 20^{\circ}, \mathrm{SNB}: 75^{\circ} \mathrm{IIA}: 150^{\circ}, \mathrm{U} 1$ to NA: $7 \mathrm{~mm}$, $\mathrm{L} 1$ to NB: $6 \mathrm{~mm}, \mathrm{~L} 1$ to $\mathrm{NB}: 10^{\circ}$
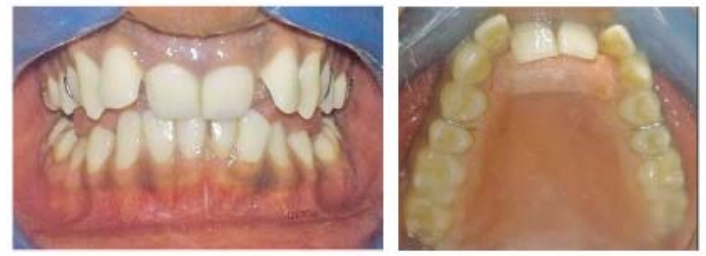

Fig-5 Upper removable appliance with anterior bite plane
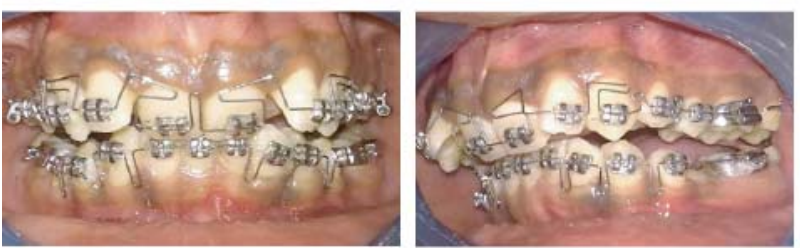

Fig-6 Initial alignment and levelling

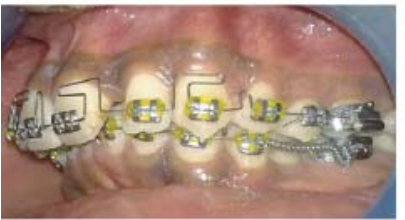

Fig-7 Open coil spring to create space for lower 2nd premolar
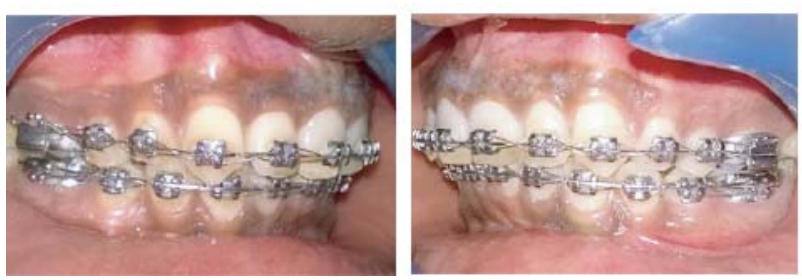

Fig-9 Arch co-ordination and Finishing
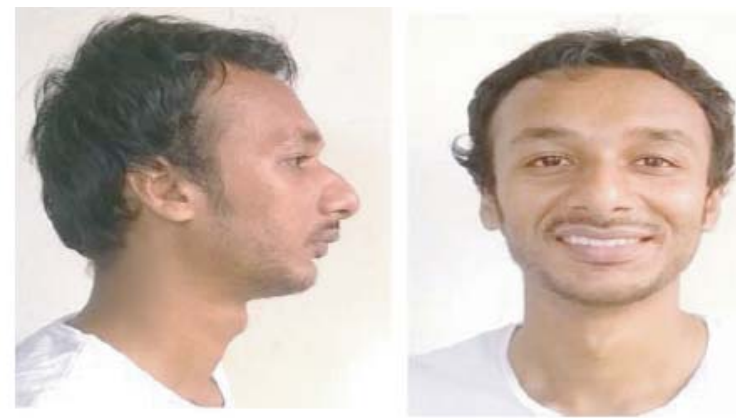

Fig-10 Post treatment extra oral photographs

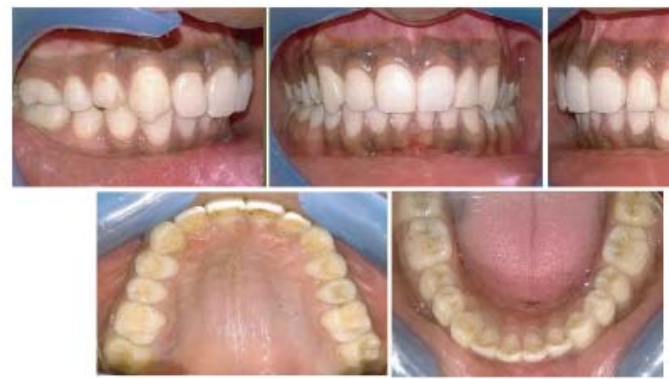

Fig-11 Post treatment intra oral photograph

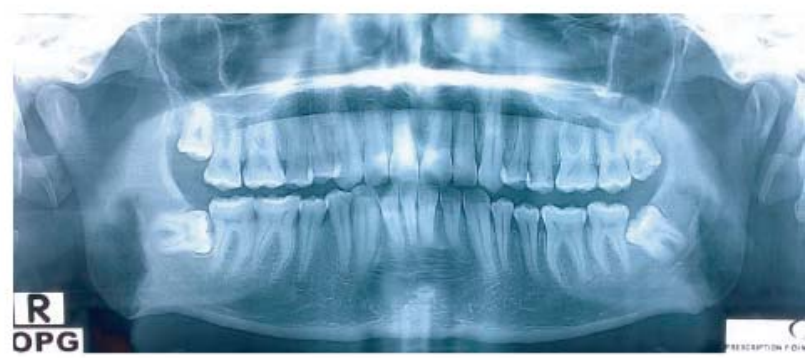

Fig-12 Post treatment Orthopentomograph (OPG)

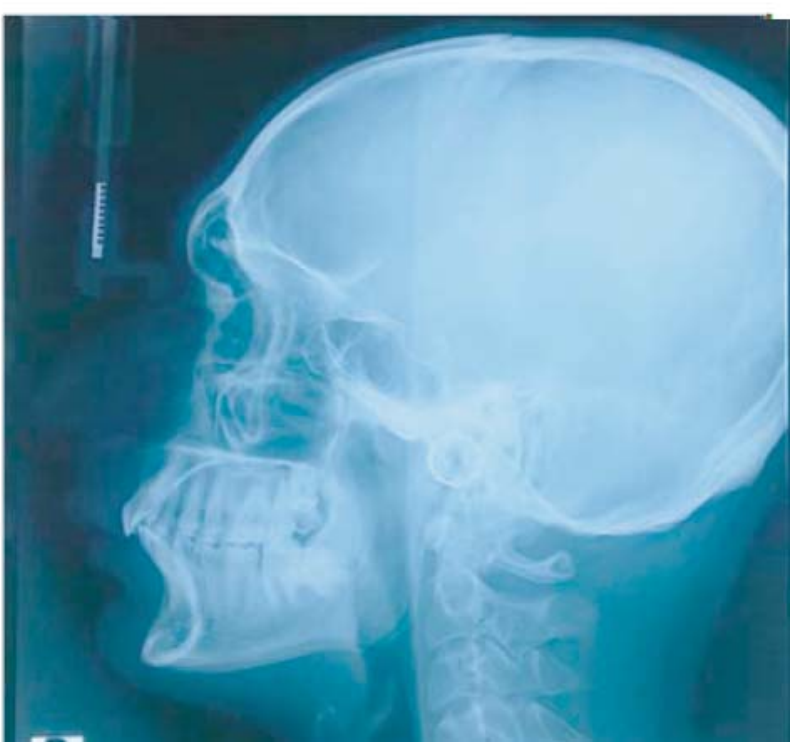

Fig-13 Post treatment Cephalometric analysis 


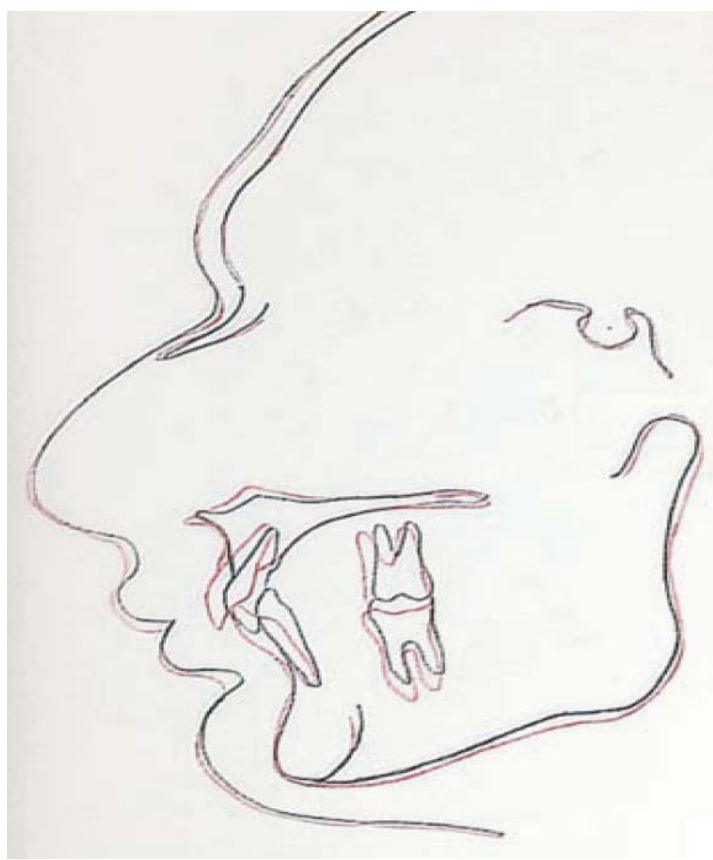

Fig-14 Total Superimposition of initial (black) and final (red) cephalometric tracings.

Table1: Pre and Post treatment Cephalometric measurements

\begin{tabular}{ccccc}
\hline Parameter & $\begin{array}{c}\text { Reference } \\
\text { Caucasian }\end{array}$ & $\begin{array}{c}\text { Reference } \\
\text { Bangladeshi }\end{array}$ & Pre tretment & Post tretment \\
\hline SNA & 82 & 83.8 & 85 & 84 \\
SNB & 80 & 81.5 & 75 & 75 \\
ANB & 2 & 22.3 & 10 & 9 \\
IIA & 131 & 117.7 & 150 & 127 \\
MPA & 32 & 25.8 & 24 & 27 \\
U1 to Na & 22 & 29.8 & & 17 \\
U1 to Namm & 4 & 8 & 7 & 2 \\
L1 to NB & 25 & 30.6 & 10 & 32 \\
L1 to NB mm & 4 & 8 & 6 & 10 \\
\hline
\end{tabular}

\section{RESULTS}

Over bite was improve from $6 \mathrm{~mm}$ to $3 \mathrm{~mm}$, and over jet also improve from $1 \mathrm{~mm}$ to $3 \mathrm{~mm}$. Satisfactory interdigitation and detailing occlusion was obtained. The periodontal tissues remained healthy during and after treatment. Maxillary arch form was also changed from squarish to oval arch form.
Patient's smile aesthetic was improved satisfactory. Post treatment orthopantomograph showed that minimal root resorption and root parallelism was satisfactory. Cephalometric evaluation showed that improved interincisal angle, ANB angle and mandibular plane angle (fig-10-14), Table 1.

\section{DISCUSSION}

Non extraction treatment of Class II, division 2 malocclusion is an effective treatment choice for the malocclusion with Bolton discrepancy in lower jaw. However several factors must be considered before making the final treatment decision. In addition, evaluation of a diagnostic wax set-up will allow the orthodontist to predict the success of the proposed treatment plan. ${ }^{7}$

\section{CONCLUSION}

Non extraction treatment of Class II division 2 malocclusion is an effective treatment choice for the malocclusion with Bolton discrepancy in lower jaw. However several factors must be considered before making the final treatment decision. In addition, evaluation of a diagnostic wax set-up will allow the orthodontist to predict the success of the proposed treatment plan.

\section{REFERENCES}

1. Martyn T. Cobourne, Padhraig S. Fleming, Andrew T. DiBiase, Sofia Ahmad, management of class II div 2 malocclusion.

2. Om.P. Kharbanda, Orthodontics; Diagnosis and management of Malocclusion and Dentofacial Deformities, 2013, page; 539.

3. McNamara, J.A.: Components of Class II malocclusion in children 8-10 years of age, Angle Orthod. 51:177; 202, 1981.

4. Kuhlberg, A. and Glynn, E.: Treatment planning considerations for adult patients, Dent. Clin. N. Am. 41:17 28, 1997.

5. Dr Situ L Shrestha,1 Dr Supreeth S Manipal,2 Dr Bikash V Shrestha,3 Dr Alok K Jaiswal4, Correction of Class II Division 2 Deepbite Malocclusion with Non - extraction Therapy

6. Nanda, R.: Biomechanics and Esthetic Strategies in Clinical Orthodontics, ed. R. Nanda, in press,W.B. Saunders Co., Philadelphia.

7. Burzin, J. and Nanda, R.: The stability of deep overbite correction, in Retention and Stability in Orthodontics, ed. R. Nanda and C.J. Burstone, W.B. Saunders, Philadelphia, 1993, p. 61.

\section{Correspondence}

Dr. Sharmin Sultana BDS, FCPS part II trainee,

Dept. of Orththodontics and Dentofacial Orthopedics,

Dhaka Dental College and Hospital.

Mob: +88 01920070664

E-mail: sharminkhulna@yahoo.com 